\title{
Optimizing the Design of a Route in Terminal Maneuvering Area Using Branch and Bound
}

\author{
Jun Zhou, Sonia Cafieri, Daniel Delahaye, and Mohammed Sbihi
}

\begin{abstract}
The sharp increase in air traffic flow causes traffic congestion in airspaces near airports, called Terminal Maneuvering Areas (TMA). The departure and arrival traffic of airports follow pre-designed routes named Standard Instrument Departure (SID) routes and Standard Terminal Arrival Routes (STAR). Optimizing these routes is crucial to regulate air traffic. Currently, SIDs and STARs are designed manually, based on the airport layout and nearby constraints. The objective of this research is to propose a methodology for designing an arrival/departure route in TMA, taking into account some constraints including obstacle avoidance. The shape of a route in horizontal plan is a succession of arcs of circles and segments. The originality of our study is, on the one hand, that the horizontal route is associated with a cone in vertical plan enveloping all ascent (or descent) aircraft profiles, and on the other hand, a branching strategy in a Branch and Bound $(B \& B)$ framework tailored on the problem is proposed.
\end{abstract}

Key words: TMA, SID/STAR design, obstacle avoidance, modeling, Branch and Bound

Jun Zhou

ENAC, Toulouse, France e-mail: junzhou@ recherche.enac.fr

Sonia Cafieri

ENAC, Toulouse, France e-mail: sonia.cafieri@enac.fr

Daniel Delahaye

ENAC, Toulouse, France e-mail: daniel.delahaye@enac.fr

Mohammed Sbihi

ENAC, Toulouse, France e-mail: mohammed.sbihi@enac.fr 


\section{Introduction}

The continuously increase in air traffic flow density causes traffic congestion in the areas surrounding airports, thus affecting the normal operation of air traffic. An area surrounding one or more neighboring airports is called Terminal Maneuvering Area (TMA), and it is designed to handle aircraft arriving to and departing from the airports. Optimizing departure and arrival procedures in TMA is therefore crucial to regulate air traffic flows. Most of the airports have pre-designed procedures indicating how aircraft depart from or arrive to airports. These procedures are called Standard Instrument Departure (SID) routes and Standard Terminal Arrival Route (STAR). A SID is a flight route followed by aircraft after take-off from an airport until the start of en-route phase. A STAR is a route which connects the last en-route way-point to the Initial Approach Fix. Currently, SID/STARs are designed manually according to operational requirements (ICAO Doc 8168), taking into account airport layout and nearby constraints. However, this kind of design is generally not very efficient and not expected to optimize any specific criterion. The objective of this work is to automatically design SID/STARs in 3D with respect to certain optimization criteria. Being this study at a strategic level, only static obstacles are taken into account. Rather than considering an individual flight, we deal with flows of flights, that is to say the flights following the same SID (or STAR) belong to the same flow.

The considered problem is in the framework of path planning. Specifically, it is a route design problem: as contrarily to trajectory design, we aim at designing routes that are not associated to the notion of time. The problem of path planning has been studied since 1980s in the robotic domain [3] [9]. Nowadays planning optimal aircraft paths becomes a rich and dynamic research area, some approaches have been summarized in [1]. In particular, computing the shortest path between two points, given a number of obstacles, is one of the most extensively studied topic [5] [8] [10] [7] [4]. Most of these works search for the shortest path in a horizontal plan, while in our study we consider searching for the shortest path in 3D. Moreover, in several works the obstacle is modeled as polygon [5] [8] [10], and in a few works as circle [7] [4]. In the present study we model the obstacle as cylinder in 3D and the projection to the horizontal plan is in form of circle.

Specifically in aircraft paths designing domain, even though there is a large number of researches, routes design in TMA is a particular problem for which to our knowledge there is not a rich literature. In [6] the author designs terminal routes getting around obstacles with a modified $\mathrm{A}^{*}$ algorithm. In our study, we design a route not only getting around obstacles, but also allowing level flights. Indeed, imposing a level flight in vertical plan is also an effective way to avoid obstacles, as it enriches the possible maneuvers and corresponds to what is done in the reality in a TMA. The different ways to avoid obstacles allow us to define specific branching strategies in a Branch and Bound tailored on the problem, that is another contribution of this work.

This paper is organized as follows. Section 2 introduces the route and obstacles modeling. Section 3 presents the proposed approach to solve the problem. Section 4 
gives some preliminary simulation results. Finally, Section 5 draws conclusions and proposes future directions.

\section{Problem Modeling}

TMA is one of the most complex types of airspace. Many constraints have to be satisfied, falling into two categories: operational constraints related to air traffic operations (such as obstacle avoidance and flyable routes), and environmental constraints (such as noise abatement). SID/STARs are designed to satisfy these constraints and to deal with the dense traffic converging to and diverging from airports. The constraints in TMA make the SID/STARs design a very complex problem. Therefore in this study we consider the simpler subproblem of designing a single route avoiding obstacles and satisfying some other operational constraints. The obstacles in TMA could be mountains, cities, military area, etc. In the following, we present the way we model routes and obstacles.

A 3D route $\gamma$ is defined by two elements: a curve $\gamma_{H}$ in a horizontal plan which is composed by a succession of arcs of circles (to bypass obstacles) and segments (to connect tangentially two arcs); a cone $\gamma_{V}$ in a vertical plan that contains all ascent (or descent) profiles of the aircraft flying on this route. The cone is defined by two straight lines whose slopes are the minimum and maximum values of the take-off (or landing) rate of the aircraft on this route. The idea of taking a cone that contains all vertical profiles is inspired by the behavior illustrated in Fig. 1, which shows some real take-off data in runway 08L of Paris CDG airport. From the figure we can see clearly that the vertical profiles are contained in a cone defined by two straight lines. The vertical profiles for landing are similar. This behavior is mainly due to the different aircraft mass and performance and to the effect of the wind.

In a horizontal plan, we define a starting point $A\left(x_{A}, y_{A}\right)$ and an ending point $B\left(x_{B}, y_{B}\right)$. In a SID case, the starting point is at the runway threshold and the ending point is an exit point of a TMA. In a STAR case, the starting point is an entry point

Fig. 1 Take-off Profiles in CDG Airport

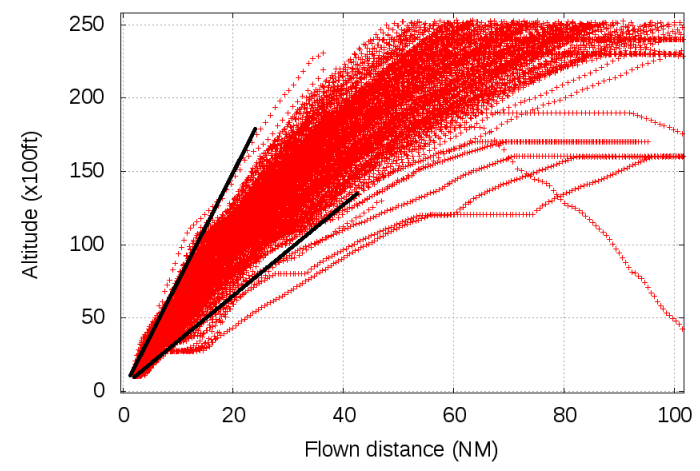



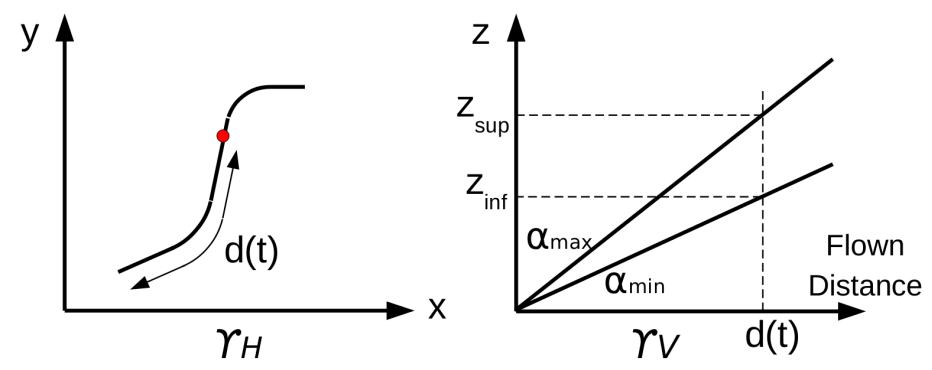

Fig. 2 An Example of $\gamma_{H}$ and $\gamma_{V}$

of a TMA and the ending point is the Final Approach Fix (FAF). The horizontal route $\gamma_{H}$ is a smooth mapping defined as:

$$
\gamma_{H}:[0,1] \rightarrow \mathbb{R}^{2}
$$

where $\gamma_{H}(0)=\left(x_{A}, y_{A}\right)$ and $\gamma_{H}(1)=\left(x_{B}, y_{B}\right)$. In a vertical plan, $\gamma_{V}$ is defined as:

$$
\begin{aligned}
\gamma_{V}:[0,1] & \rightarrow I^{\mathbb{R}} \\
t & \rightarrow\left[z_{\text {inf }}(d(t)), z_{\text {sup }}(d(t))\right]
\end{aligned}
$$

where $I^{\mathbb{R}}$ defines the set of intervals of $\mathbb{R}$, and $d(t)=\int_{0}^{t}\left\|\gamma_{H}^{\prime}(s)\right\|_{2} \mathrm{~d} s$ is the flown distance until $t$ in horizontal plan, $\left[z_{\text {inf }}(d), z_{\text {sup }}(d)\right]$ is the interval defined by the cross section of the cone at $d$. The vertical profile $\gamma_{V}$ is associated to the horizontal curve $\gamma_{H}$ through the flown distance $d$. Figure 2 illustrates an example of how $\gamma_{H}$ is associated with $\gamma_{V}$ in the case of a SID, where $\alpha_{\min }$ and $\alpha_{\max }$ are the minimum and maximum values of take-off rate of aircraft on this route.

In the case of a SID, the starting point $A$ is associated with an altitude $z_{A}$; the ending point $B$ is associated with an altitude interval $\left[z_{B}, \overline{z_{B}}\right]$ which indicates the altitude interval to exit TMA. Therefore the boundary conditions are $\gamma_{V}(0)=\left[z_{A}, z_{A}\right]$ and $\gamma_{V}(1) \subset\left[z_{B}, \overline{z_{B}}\right]$. Similarly, in the case of a STAR, the altitude interval of the starting point $A$ is $\left[\underline{z_{A}}, \overline{z_{A}}\right]$ which indicates the interval to enter TMA; the altitude of the ending point $B$ is $z_{B}$. The boundaries conditions are: $\gamma_{V}(0)=\left[z_{A}, \overline{z_{A}}\right]$ and $\gamma_{V}(1)=$ $\left[z_{B}, z_{B}\right]$. Note that, in order to guarantee that any SID reaches $B$ within $\left[\underline{z_{B}}, \overline{z_{B}}\right]$, we set $z_{B}=z_{A}+\alpha_{\min } \cdot d(A, B)$, where $\alpha_{\min }$ is the minimum taking-off slope, and $d(A, B)$ is the Euclidean distance between the starting and ending points $A$ and $B$. Similarly, to guarantee that any STAR reaches $z_{B}$ at FAF, we set $z_{A}=z_{B}+\alpha_{\text {min }} \cdot d(A, B)$ and $\overline{z_{A}}=$ $z_{B}+\alpha_{\max } \cdot d(A, B)$, where $\alpha_{\min }\left(\alpha_{\max }\right)$ is the minimum (maximum) landing slope. A level flight can be imposed to ensure that $\overline{z_{B}}$ (respectively, $z_{B}$ ) is not exceeded in the case of a SID (respectively, STAR).

The obstacles, together with their protection areas, in number of $m \in N$, are modeled as cylinders in 3D, whose bases are parallel to the horizontal plan as presented in Fig. 3. Each cylinder $\Omega_{i}, i=1, \cdots, m$ is defined by $\left(C_{i}\left(x_{i}, y_{i}\right), r_{i}, z_{i_{\text {inf }}}, z_{i_{\text {sup }}}\right)$, where $C_{i}\left(x_{i}, y_{i}\right)$ and $r_{i}$ are the center and the radius of the two bases respectively; $z_{i n f}$ and $z_{i s u p}$ are the altitude of the lower and upper bases. These obstacles are numbered in 
Fig. 3 Obstacle Modelization

Fig. 4 Obstacles Numbering
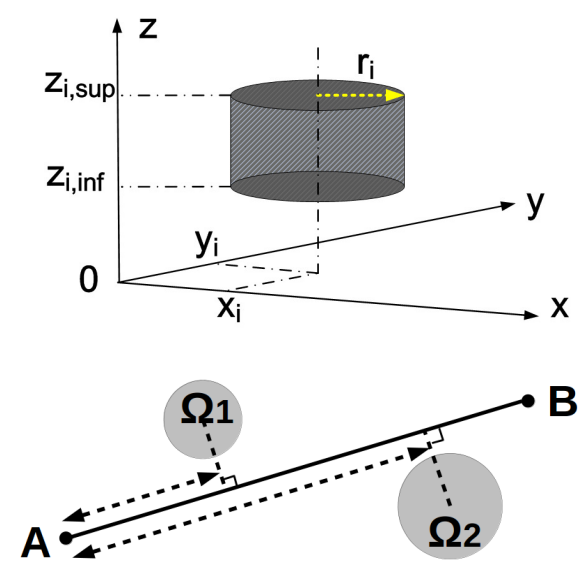

an increasing order of length $\left(A, \operatorname{Proj}_{(A B)} C_{i}\right)$ where $\operatorname{Proj}_{(A B)} C_{i}$ is the projection of $C_{i}$ onto the line $(A B)$. An illustration is presented in Fig. 4.

Let us define an active obstacle as an obstacle that is touched by a route and has to be avoided according to one of the following maneuvers: turn counter-clockwise, turn clockwise or impose a level flight. Each cylinder $\Omega_{i}$ is associated with two decision variables $s_{i}$ and $t_{i}: s_{i}$ defines whether $\Omega_{i}$ is active or not:

$$
s_{i}=\left\{\begin{array}{l}
0, \text { if } \Omega_{i} \text { not active } \\
1, \text { if } \Omega_{i} \text { active }
\end{array}\right.
$$

while $t_{i}$ defines the ways an active obstacle $\Omega_{i}$ is avoided:

$$
t_{i}=\left\{\begin{array}{l}
0, \text { if turn counter-clockwise } \\
1, \text { if turn clockwise } \\
2, \text { if impose level flight }
\end{array}\right.
$$

Once the values of decision variables are chosen, the horizontal route is computed by connecting tangentially the successive active $\left(s_{i}=1\right)$ obstacles with $t_{i}=0$ or $t_{i}=1$ in the increasing order of their numbering. Active obstacles with $t_{i}=0$ are bypassed counter-clockwise and those with $t_{i}=1$ are bypassed clockwise. This horizontal route is hence built piecewise: it is composed by $\left(\sum_{i=1}^{m} s_{i}-\sum_{i=1}^{m} \max \left(t_{i}-\right.\right.$ $1,0)+1)$ straight line segments and $\left(\sum_{i=1}^{m} s_{i}-\sum_{i=1}^{m} \max \left(t_{i}-1,0\right)\right)$ arcs of circles. Routes are therefore constrained to lie on the border of obstacles. In order to ensure flyable routes, the radius of the arcs of circles is imposed to be at least equal to $3 \mathrm{Nm}$ (FAA Orders 8260.54A and 8260.58). Note that these arcs can be followed using a type of Performance Based Navigation (PBN) named Required Navigation Performance (RNP). Then, a vertical profile is associated to the horizontal route, taking into account $\alpha_{\min }, \alpha_{\max }$, and imposing a level flight below (respectively above) the active obstacle $\Omega_{i}$ in a SID (respectively STAR) case when $t_{i}=2$. If some active obstacles with $t_{i}=2$ is not intersected by the cone associated with the horizontal 


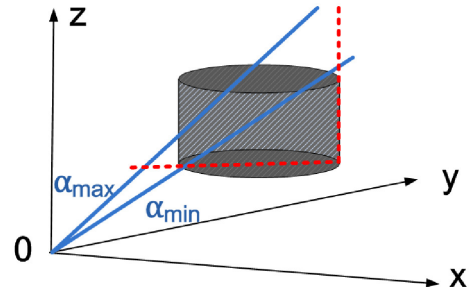

a

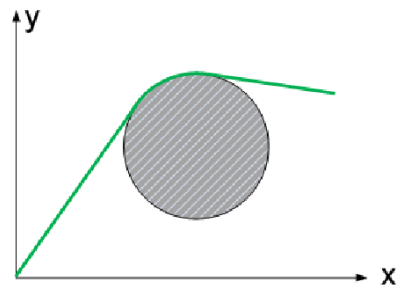

$\mathrm{C}$

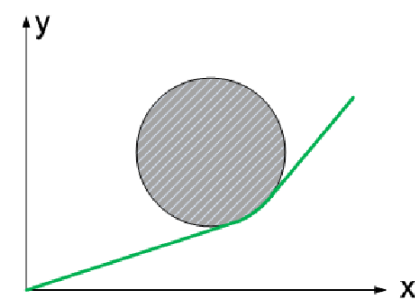

b

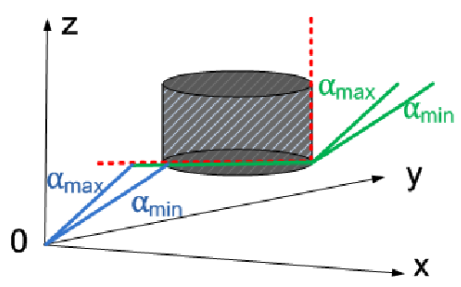

d

Fig. 5 The Routes Associated to Different Values of the Decision Variables. a $s_{i}=0$, 3D View. b $\left(s_{i}, t_{i}\right)=(1,0), 2 \mathrm{D}$ View. $\mathbf{c}\left(s_{i}, t_{i}\right)=(1,1), 2 \mathrm{D}$ View. $\mathbf{d}\left(s_{i}, t_{i}\right)=(1,2), 3 \mathrm{D}$ View

route, then the route is unfeasible regarding to our definition of "active obstacle". Note that the way of building a horizontal route simplifies the computation but it does not necessarily lead to the shortest horizontal route between $A$ and $B$.

An illustration of different values of the decision variables for an example of a SID in a TMA with one obstacle is presented in Fig. 5. In Fig. 5a, the obstacle is not active, so $s_{i}=0$. The horizontal route is a straight line segment connecting $A$ and $B$. It is associated with a cone in the vertical plan. This route in the considered example is not a feasible one, because it intersects the obstacle. Then when the obstacle is active $\left(s_{i}=1\right), 3$ possibilities are considered to avoid it (Figs. $5 \mathrm{~b}, 5 \mathrm{c}, 5 \mathrm{~d}$ ): turn counter-clockwise, turn clockwise and impose a level flight under the obstacle at altitude $z_{i n f}$, corresponding to $t_{i}=0,1,2$ respectively.

Two examples of SIDs are presented in Fig. 6 showing how a route is computed, given the values of the decision variables, in the case with more than one obstacle. In example 1 (Figs. 6a, 6b), given $\left(s_{1}, t_{1}\right)=(1,0),\left(s_{2}, t_{2}\right)=(1,1)$, the horizontal route is composed by five parts: three segments and two arcs of circles. The three segments are used to connect tangentially the starting point to $\Omega_{1}, \Omega_{1}$ to $\Omega_{2}$ and $\Omega_{2}$ to the ending point respectively. The two arcs are used to bypass $\Omega_{1}$ counter-clockwise and $\Omega_{2}$ clockwise respectively. In example 2 (Figs. 6c, 6d), given $\left(s_{1}, t_{1}\right)=(1,2),\left(s_{2}, t_{2}\right)=(1,1)$, the horizontal route is constructed by only bypassing $\Omega_{2}$, thus it is composed by two segments and one arc of circle. In vertical plan, when the route reaches the altitude of the lower basis of $\Omega_{1}$, a level flight is im- 


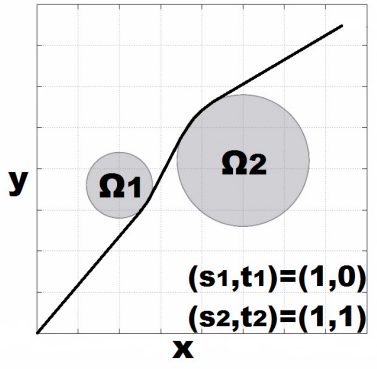

a

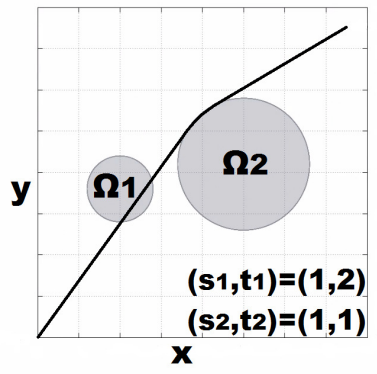

c

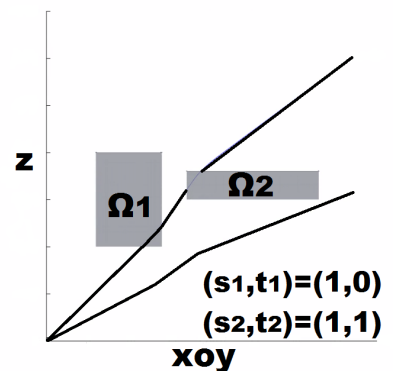

b

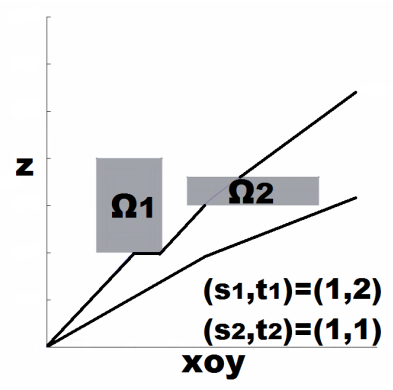

d

Fig. 6 Routes Construction. a Example 1: Horizontal Plan. b Example 1: Vertical Plan. c Example 2: Horizontal Plan. d Example 2: Vertical Plan. Note that in $\mathbf{b}$ and d, slopes appear discontinuous as an effect of a projection of a 3D image on a plan

posed. The level flight ends at the flown distance where the horizontal route passes the boundary of $\Omega_{1}$ and is no more intersected by $\Omega_{1}$.

Besides obstacle avoidance, further constraints are related to level flights. First, the number of level flights is bounded by a maximum number $N_{\max }$, usually fixed to 2, for each route:

$$
\sum_{i=1}^{m} \max \left(t_{i}-1,0\right) \leq N_{\max }
$$

Second, as the altitudes of imposed level flights have a direct impact on the noise pollution, a minimum altitude $H_{\min }$ for each level flight is defined. In practice, we impose the following constraints: in a SID case (respectively a STAR case), for an obstacle $\Omega_{i}$, if $z_{i \text { inf }}<H_{\text {min }}$ (respectively $z_{\text {sup }}<H_{\text {min }}$ ), then no level flight is imposed below (respectively above) it, therefore $t_{i} \in\{0,1\}$.

Third, as to take into account the passengers comfort, the length of each level flight should not be too short, a minimum length $L_{\min }$ for each level flight is imposed.

We minimize a weighted sum $L_{\gamma}$ of the length of the route $\gamma$ in the horizontal plan and the length related to the level flights. More precisely: 
Fig. 7 Branch and Bound Branching Strategy

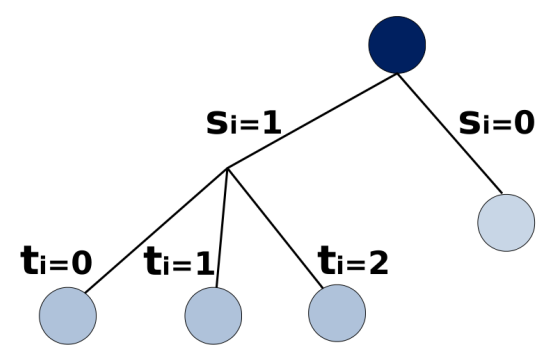

$$
L_{\gamma}=c_{1} \times\left(\int_{0}^{1}\left\|\gamma_{H}^{\prime}(t)\right\|_{2} \mathrm{~d} t\right)+c_{2} \times L_{\min } \times \sum_{i=1}^{m} \max \left(t_{i}-1,0\right)
$$

The coefficients $c_{1}$ and $c_{2}$ are two penalty parameters, their values depend on the importance of the corresponding term. The obtained problem (denoted by $(\mathscr{P})$ ) is a combinatorial optimization problem. In the next section we explain the proposed solution approach for this problem.

\section{Solution Approach: Branch and Bound}

To solve the problem, we apply a Branch and Bound method. In [2], a path planning problem avoiding circular obstacles is studied in 2D. A branching strategy is proposed, where, for each obstacle, two branches are created depending on the clockwise or counter-clockwise obstacle bypassing. We extend this branching strategy to take into account the specificity of our problem, where obstacles can be avoided also by imposing a level flight below (SID case) or above (STAR case) the obstacle. Our branching strategy is illustrated in Fig. 7 . We start by setting $\Omega_{i}$ as active $\left(s_{i}=1\right)$ or not $\left(s_{i}=0\right)$; when it is active, we develop three branches in order to account for the 3 possibilities of avoiding it: counter-clockwise $\left(t_{i}=0\right)$, clockwise $\left(t_{i}=1\right)$ or imposing a level flight $\left(t_{i}=2\right)$.

The lower bound for each subproblem is computed by generating the route which bypasses only the active obstacles and by calculating its length according to the objective function (6). The obtained lower bound is then used to identify whether a branch requires further subdivisions.

We present a step-by-step illustration (Fig. 8) to show how the Branch and Bound method works. The starting and ending points as well as two obstacles $\Omega_{1}, \Omega_{2}$ are presented in Fig. 8 a. We take $c_{1}=1, c_{2}=1$, that is to say we penalize the length of level flights in the objective function.

Step 1: We develop 4 branches on $\Omega_{1}$. We start by deviating the route counterclockwise, and we obtain a route that does not intersect $\Omega_{2}$. The lower bound in this case is $100320 \mathrm{~m}$. Besides, the value of the objective function associated with 
solution $\left(s_{1}, t_{1}\right)=(1,0), s_{2}=0$ is equal to the lower bound. Therefore, no further exploration is needed.

Step 2: Another branch on $\Omega_{1}$ is developed by deviating the direct route clockwise around $\Omega_{1}$. The length of this horizontal route is the lower bound of this subproblem, the value is greater than the current best value. There is no possibility to get a better solution by further branching on $\Omega_{2}$, therefore we cut this branch.

Step 3: The third branch on $\Omega_{1}$ is obtained by imposing a level flight, as shown in Figs. 8d, 8e. The lower bound is greater than the current best value, so the branch is cut.

Step 4: The last branch on $\Omega_{1}$ is with $s_{1}=0$. The route intersects $\Omega_{2}$ and its lower bound, which corresponds to the length of the direct route, is less than the current best length, thus 4 branches on $\Omega_{2}$ are developed. In the case $s_{2}=0$, the route is not feasible thus not accepted.

Step 5: By branching counter-clockwise around $\Omega_{2}$, the obtained route is still intersecting $\Omega_{1}$, thus it is not accepted.

Step 6: By branching clockwise around $\Omega_{2}$, we obtain a feasible route with length greater than the current best value, so it is not accepted.

Step 7: The last branch is obtained by imposing a level flight under $\Omega_{2}$ as shown in Figs. 8i, 8j. This route is still encountered by $\Omega_{1}$, so it is not accepted.

All the possible branches are considered. The best distance is $100320 \mathrm{~m}$ by taking $\left(s_{1}, t_{1}\right)=(1,0), s_{2}=0$.

The maximum size of the search space of our problem is $4^{m}$, where $m$ is the number of obstacles. Indeed we have 4 possibilities to deal with each obstacle. The size of the search space can be reduced significantly by applying the pre-processing techniques developed in [4]. In fact, the authors prove firstly that the shortest path connecting two points and avoiding circular obstacles must lie into an ellipse containing these two points. Moreover, they prove that the shortest path lies in a convex hull of a few circular obstacles around the line segment connecting the starting and ending points. We apply these pre-processing techniques to reduce the number of considered obstacles before applying our approach. These filters still hold when considering cylindrical obstacles. A 3-dimensional feasible route can be indeed built based on the 2D shortest path avoiding the obstacle projections on the plan. Some simulation results with and without the pre-processing techniques are presented in Sect. 4.

\section{Simulation Results}

In this section we present two simulation examples. The first one is the case of a SID, the second one is the case of a STAR. Tests were run on a Linux platform with a $2.4 \mathrm{GHz}$ processor and $8 \mathrm{~GB}$ RAM.

Different strategies are applied to choose the next sub-problem to solve and the next obstacle to branch on. The simulation results presented in this section use the Best Lower Bound (BLB) for selecting the next sub-problem, and use the First In- 


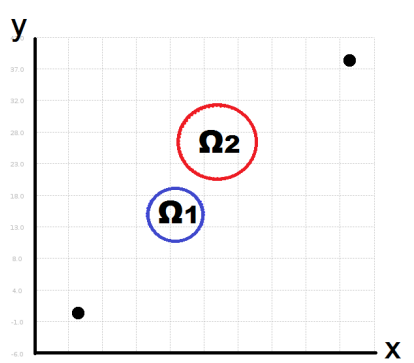

a

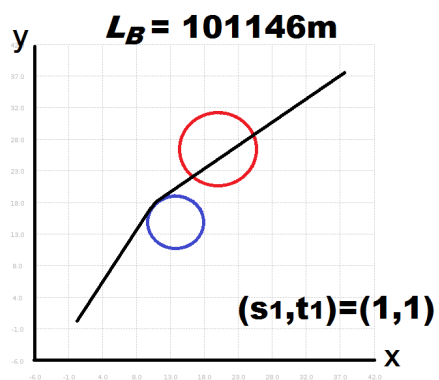

$\mathrm{c}$

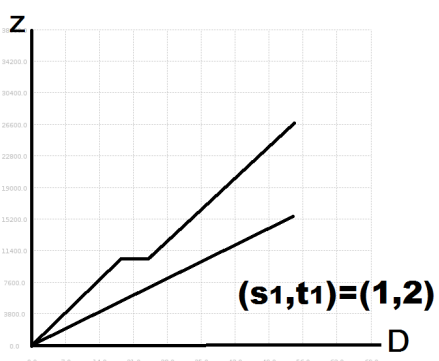

e

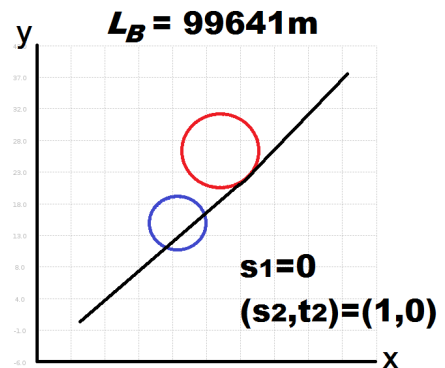

$\mathrm{g}$

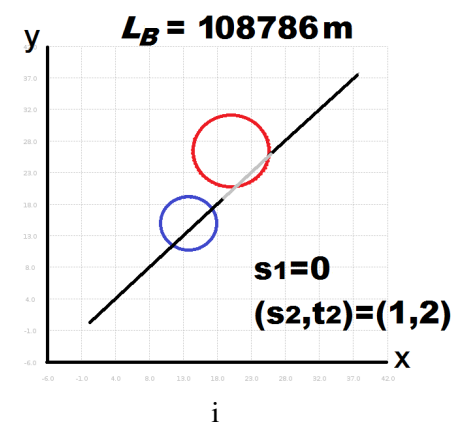

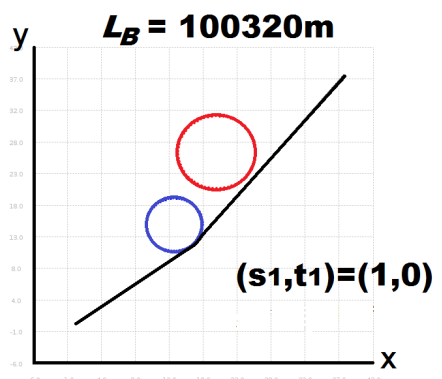

b

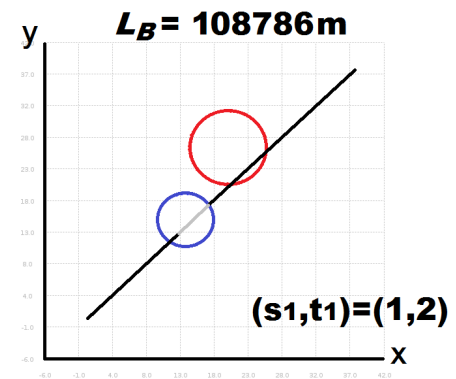

d

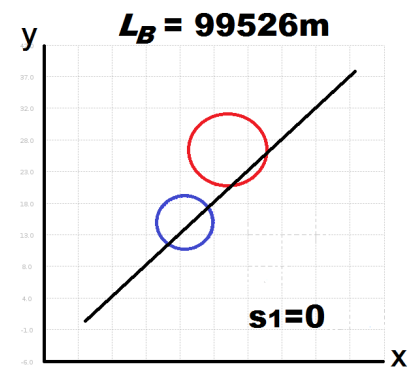

f

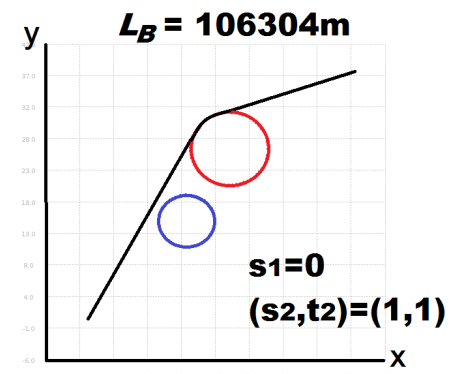

$\mathrm{h}$

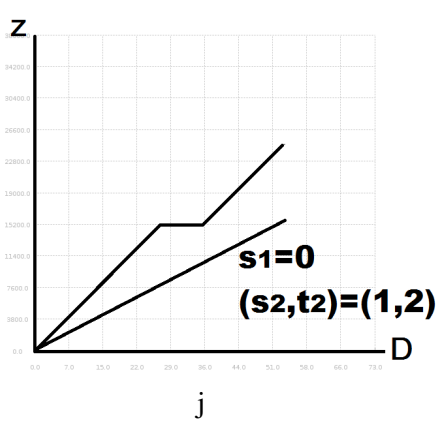

Fig. 8 Branch and Bound Illustration. a Step 0. b Step 1. c Step 2. d Step 3, Horizontal Plan. e Step 3, Vertical Plan. f Step 4. g Step 5. h Step 6. i Step 7, Horizontal Plan. j Step 7, Vertical Plan 
tersected Obstacle (FIO) for selecting the next obstacle to branch on. In fact, the combination of strategies "BLB+FIO" gives the minimum computing time together with the minimum number of iterations in most of the tests.

In the first example, the input data are (unit in meter):

- Starting point $A,\left(x_{A}, y_{A}, z_{A}\right)=(0,0,0)$

- Ending point $B,\left(x_{B}, y_{B},\left[\underline{z_{B}}, \overline{z_{B}}\right]\right)=(70376,70376,[4777,9754])$

- $\alpha_{\text {min }}=4.8 \%, \alpha_{\max }=9.2 \%$

- $N_{\max }=2, L_{\min }=9260, H_{\min }=914$

There are nine obstacles. Table 1 gives the center $\left(x_{i}, y_{i}\right)$, radius $r_{i}$ and altitudes of the two bases $\left(z_{i_{\text {inf }}}, z_{i_{\text {sup }}}\right)$ of obstacle $\Omega_{i}, i=1, \cdots, 9$. The unit is in meter. The simulation results are presented in Table 2 and Fig. 9. We carried out two tests with different values of $c_{1}, c_{2}$ : the first one with $c_{1}=1, c_{2}=0$; the second one with $c_{1}=1, c_{2}=1$. When $c_{1}=1, c_{2}=0$, the length of level flights is not penalized in the objective function, thus Figs. 9a, 9b show that the optimal route is obtained with a level flight to avoid the obstacle with the center coordinates $(29632,25928)$. However, when $c_{1}=1, c_{2}=1$ the length of level flight is penalized in the objective function, and as a result a counter-clockwise turn is made to avoid the mentioned obstacle instead of using a level flight, as shown in Figs. 9c, 9d. Moreover, thanks to the ellipse and convex hull filters, which reduces the number of the potential obstacles from 9 to 5 , the computation time and the number of iterations are reduced effectively.

In the second example, the input data are (unit in meter):

- Starting point $A,\left(x_{A}, y_{A},\left[z_{A}, \overline{z_{A}}\right]\right)=(0,0,[4454,7722])$

- Ending point $B,\left(x_{B}, y_{B}, z_{B}\right)=(96304,96304,914)$

Table 1 Example 1: Characteristics of Nine Obstacles

\begin{tabular}{lll}
\hline$\left(x_{i}, y_{i}\right)(\mathrm{m})$ & $r_{i}(\mathrm{~m})$ & $\left(z_{\text {inf }}, z_{\text {isup }}\right)(\mathrm{m})$ \\
\hline$(7408,14816)$ & 3704 & $(701,2377)$ \\
$(9260,35100)$ & 11112 & $(884,1707)$ \\
$(9260,57412)$ & 4630 & $(2286,4389)$ \\
$(29632,25928)$ & 9260 & $(2682,3109)$ \\
$(33336,48152)$ & 5556 & $(2835,5425)$ \\
$(42596,14816)$ & 5556 & $(2286,4389)$ \\
$(48152,51856)$ & 7408 & $(3536,6828)$ \\
$(61116,29632)$ & 9260 & $(3048,6706)$ \\
$(70376,51826)$ & 9260 & $(4154,8022)$ \\
\hline
\end{tabular}

Table 2 Example 1: Simulation Results

\begin{tabular}{llllll}
\hline \multirow{2}{*}{$\left(c_{1}, c_{2}\right)$} & \multicolumn{3}{c}{ no pre-processing } & \multicolumn{3}{c}{ with pre-processing } & \multirow{2}{*}{$L_{\gamma}(\mathrm{m})$} \\
& Time $(\mathrm{s})$ & Iterations & Time $(\mathrm{s})$ & Iterations & \\
\hline$(1,0)$ & 0.47 & 254 & 0.09 & 46 & 100082 \\
$(1,1)$ & 0.57 & 307 & 0.20 & 115 & 102478 \\
\hline
\end{tabular}



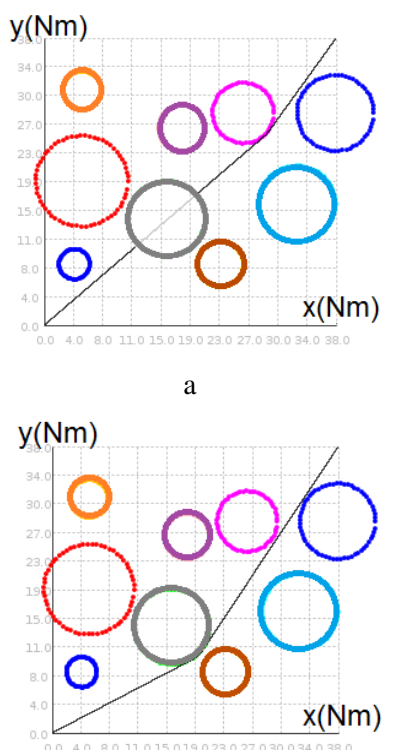

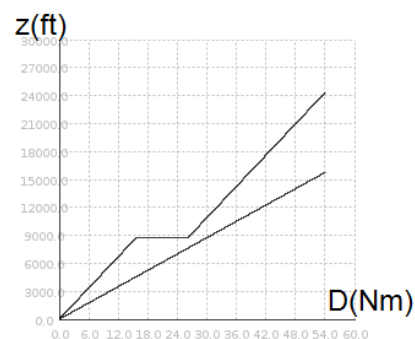

b

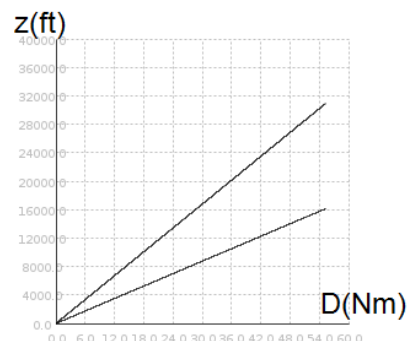

d

Fig. 9 Example 1: Simulation Results. a $\gamma_{H}, c_{1}=1, c_{2}=0 . \mathbf{b} \gamma_{V}, c_{1}=1, c_{2}=0 . \mathbf{c} \gamma_{H}, c_{1}=1, c_{2}=1$. d $\gamma_{V}, c_{1}=1, c_{2}=1$

- $\alpha_{\min }=2.6 \%, \alpha_{\max }=5 \%$

- $N_{\max }=2, L_{\min }=9260, H_{\min }=914$

Table 3 Example 2: Characteristics of Seven Obstacles

\begin{tabular}{lll}
\hline$\left(x_{i}, y_{i}\right)(\mathrm{m})$ & $r_{i}(\mathrm{~m})$ & $\left(z_{\text {inf }}, z_{i_{\text {sup }}}\right)(\mathrm{m})$ \\
\hline$(22224,22224)$ & 9260 & $(0,5486)$ \\
$(27780,74080)$ & 14816 & $(0,3048)$ \\
$(48152,96304)$ & 7408 & $(0,4572)$ \\
$(59264,51856)$ & 12964 & $(0,4572)$ \\
$(72228,24076)$ & 9260 & $(0,4572)$ \\
$(77784,81488)$ & 14816 & $(0,3048)$ \\
$(96304,48152)$ & 12964 & $(0,4572)$ \\
\hline
\end{tabular}

There are seven obstacles defined by the parameters presented in Table 3 . The simulation results are presented in Table 4 and Fig. 10. Note that the optimal route in the case with $c_{1}=1, c_{2}=0$ is the same as the one in the case with $c_{1}=1, c_{2}=$ 1. Even though by taking $c_{2}=0$, level flights are not penalized in the objective function, no level flight is imposed in the optimal solution. The reason is that the routes with level flights do not offer a better value of the objective function or do 
Table 4 Example 2: Simulation Results

\begin{tabular}{llllll}
\hline \multirow{2}{*}{$\left(c_{1}, c_{2}\right)$} & \multicolumn{3}{c}{ no pre-processing } & \multicolumn{3}{c}{ with pre-processing } & \multirow{2}{*}{$L_{\gamma}(\mathrm{m})$} \\
& Time $(\mathrm{s})$ & Iterations & Time $(\mathrm{s})$ & Iterations & \\
\hline$(1,0)$ & 0.64 & 349 & 0.09 & 45 & 142230 \\
$(1,1)$ & 0.36 & 188 & 0.09 & 45 & 142230 \\
\hline
\end{tabular}

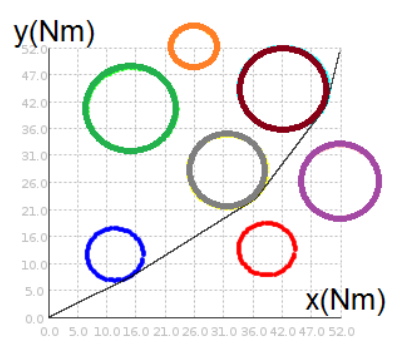

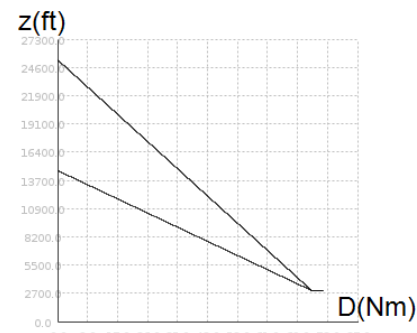

b

Fig. 10 Example 2: Simulation Results. a $\gamma_{H}, c_{1}=1, c_{2}=0$ or $c_{1}=1, c_{2}=1$. b $\gamma_{V}, c_{1}=1, c_{2}=0$ or $c_{1}=1, c_{2}=1$

not satisfy the constraints that mentioned before. By applying the pre-processing techniques, the number of the potential obstacles is reduced from 7 to 3 , therefore the computation time and the number of iterations are reduced significantly.

\section{Conclusion and Perspectives}

In this paper, we introduce a methodology for generating a $3 \mathrm{D}$ route in TMA at strategic level, performed by a deterministic global optimization approach. The route is represented by a horizontal curve associated to a cone in the vertical plan. We develop three ways to avoid an obstacle: turn clockwise or counter-clockwise (2D) and level flight (3D) which correspond to branching strategies in a Branch and Bound tailored on the problem. By setting appropriately the penalization coefficients, it is possible to obtain continuous and smooth routes which are available for Continuous Climb Operations (CCO) and Continuous Descent Operations (CDO). To summarize, this approach can be regarded as a decision support tool for the designing of SID/STARs.

In future work, we will consider the problem of designing several routes taking into account the avoidance of obstacles and the separation between them. One possible approach is to decompose the problem in three parts: (a) generating each route separately by applying the Branch and Bound method presented in this paper; (b) detecting the conflicts between the generated routes; (c) eliminating the conflicts by adding fictitious cylinder obstacles at the position of the conflicts. 
Acknowledgements This work has been partially supported by Civil Aviation University of China, by National Natural Science Foundation of China (NNSFC) through grant NNSFC 61201085 and by French National Research Agency (ANR) through grant ANR 12-JS02-009-01 ATOMIC. The authors would like to thank Serge Roux from the French Civil Aviation University (ENAC), for the interesting discussions and useful comments.

\section{References}

1. Delahaye, D., Puechmorel, S., Tsiotras, P., Feron, E.: Mathematical models for aircraft trajectory design: A survey. Air Traffic Management and Systems Lectures Notes in Electrical Engineering. 290, 205-247 (2014)

2. Eele, A., Richards, A.: Path planning with avoidance using nonlinear branch and bound optimization. J. Guid. Control. Dynam. 32, 384-394 (2009)

3. Gallina, P., Gasparetto, A.: A technique to analytically formulate and to solve the 2dimensional constrained trajectory planning problem for a mobile robot. J. Intell. Robotics Syst. 27, 237-262 (2000)

4. Kim, D.S., Yu, K., Cho, Y., Kim, D., Yap, C.: Shortest paths for disc obstacles. Lect. Notes Comput. Sci. 3045, 62-70 (2004)

5. Lozano-Pérez, T., Wesley, M.A.: An algorithm for planning collison-free paths among polyhedral obstacles. Commun. ACM. 22, 560-570 (1979)

6. Pfeil, D.M.: Optimization of airport terminal-area air traffic operations under uncertain weather conditions. PhD thesis, Massachusetts Institute of Technology, 2011

7. Pocchiola, M., Vegter, G.: Minimal tangent visibility graphs. Comput. Geom. Theory Appl. 6, 303-314 (1996)

8. Rohnert, H.: Shortest paths in the plane with convex polygonal obstacles. Inf. Process. Lett. 23, 71-76 (1986)

9. Souissi, O. et al.: Path planning: A 2013 survey. In: Proceedings of the 2013 International Conference on Industrial Engineering and Systems Management, pp. 1-8. Rabat (2013)

10. Storer, J.A., Reif, J.H.: Shortest paths in the plane with polygonal obstacles. J. ACM. 41, 982-1012 (1994) 